\title{
Balloon-expandable covered stent implantation for treatment of a traumatic pulmonary artery pseudoaneurysm in a pediatric patient
}

\author{
Vincent J. Palmieri, MD, M. Elisabeth Heal, DO, Shahryar M. Chowdhury, MD, and \\ Varsha M. Bandisode, MD, Charleston, SC
}

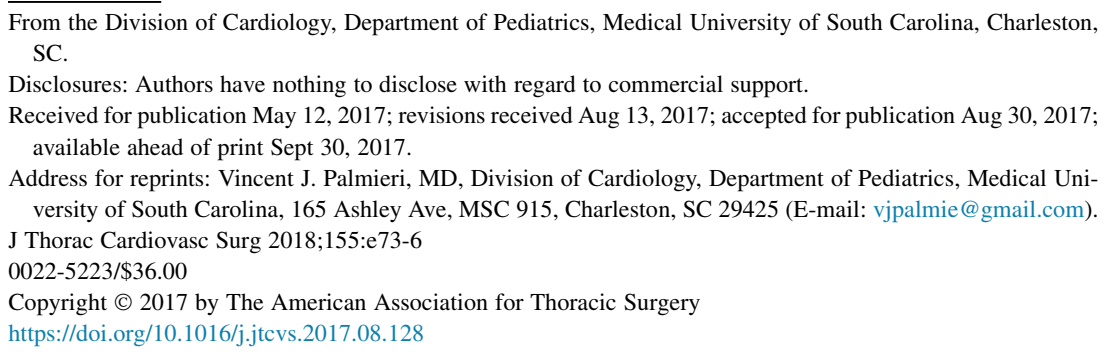

From the Division of Cardiology, Department of Pediatrics, Medical University of South Carolina, Charleston, SC.

Disclosures: Authors have nothing to disclose with regard to commercial support.

Received for publication May 12, 2017; revisions received Aug 13, 2017; accepted for publication Aug 30, 2017; available ahead of print Sept 30, 2017.

Address for reprints: Vincent J. Palmieri, MD, Division of Cardiology, Department of Pediatrics, Medical University of South Carolina, 165 Ashley Ave, MSC 915, Charleston, SC 29425 (E-mail: vjpalmie@ gmail.com). J Thorac Cardiovasc Surg 2018;155:e73-6 $0022-5223 / \$ 36.00$

Copyright (c) 2017 by The American Association for Thoracic Surgery https://doi.org/10.1016/j.jtcvs.2017.08.128

Traumatic pulmonary artery pseudoaneurysms (PAPs) are rare in the pediatric population. Although open surgical repair was once the most viable means of care, endovascular approaches have become more favorable as techniques and devices have improved. Currently, endovascular management varies, as covered stent grafts and coil embolization with and without stent coverage are among the various approaches taken to treat PAPs. ${ }^{1-4} \mathrm{~A}$ large-diameter, balloon-expandable, covered endovascular stent has recently been approved by the US Food and Drug Administration as commercially available for use in the United States. At the time of our case, the NuMED covered Cheatham platinum balloon-expandable stent (NuMED, Inc, Hopkinton, NY) was available to centers participating in the Coarctation of the Aorta Stent Trial (COAST) and the Pulmonary Artery Repair with Covered Stents (PARCS) trial. This case demonstrates the youngest reported patient to have undergone successful covered stent implantation for a PAP under emergency use guidelines, performed with permission from the US Food and Drug Administration.

\section{CLINICAL SUMMARY}

We present the case of an 8-year-old boy who was a restrained, front-seat passenger in a motor vehicle collision and was taken to an outside hospital. His initial Glasgow Coma Scale score was 6; it quickly improved to 14 to 15 , at which time he was transported to our emergency department. He was intubated for oxygen saturations in the $80 \%$ to $90 \%$ range and significant bilateral pulmonary contusions. Initial imaging demonstrated these contusions, along with posterior rib fractures (including the 4th, 5th, and 6th ribs), bilateral pneumothoraxes, a small pericardial effusion, lacerations of splenic poles, a distal femur fracture, metatarsal fractures, and a medial orbital wall fracture without intracranial injury.

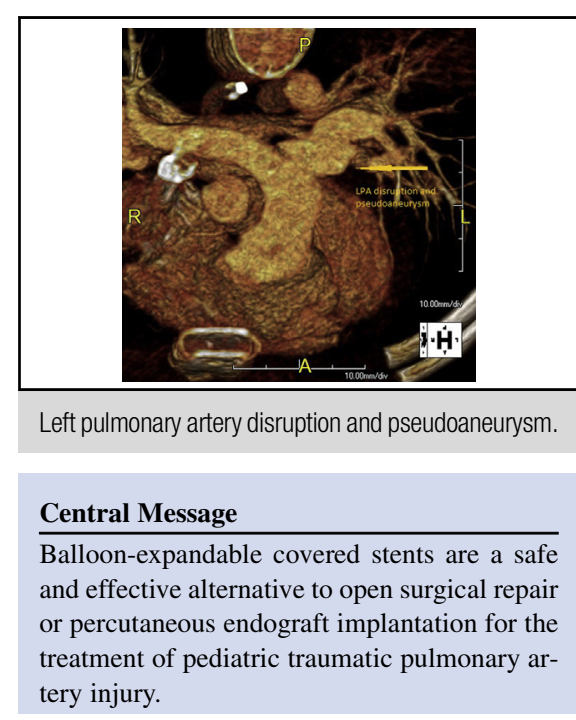

See Editorial Commentary page e77.
A follow-up gated chest computed tomographic angiogram was performed that demonstrated a $1.9 \times 1.1 \times 2.6-\mathrm{cm}$ contrast-filled outpouching, described as a moderate pseudoaneurysm at the proximal left pulmonary artery from traumatic pulmonary artery disruption (Figures 1,2, and 3). The level of the injury was just beyond the bifurcation of the pulmonary arteries and posteroinferior to the proximal left pulmonary artery, with the entry into the pseudoaneurysm in the left pulmonary artery. There was no evidence of aortic injury, but there was a small hemopericardium. The patient was started on an esmolol drip to minimize blood pressure, heart rate, and wall stress. Because of the pulmonary contusions and continual hemoptysis, the patient was deemed high risk for the systemic anticoagulation required for an open surgical repair, which was deemed necessary by the surgical team for successful repair of the left pulmonary artery. The decision was made to attempt percutaneous covered stent placement across the PAP in the pediatric cardiac catheterization laboratory to prevent further expansion and possible complete tear of the pseudoaneurysm before lifting sedation. As a participant in the multicenter PARCS trial, our institution has access to balloon-expandable Cheatham platinum covered stents. 


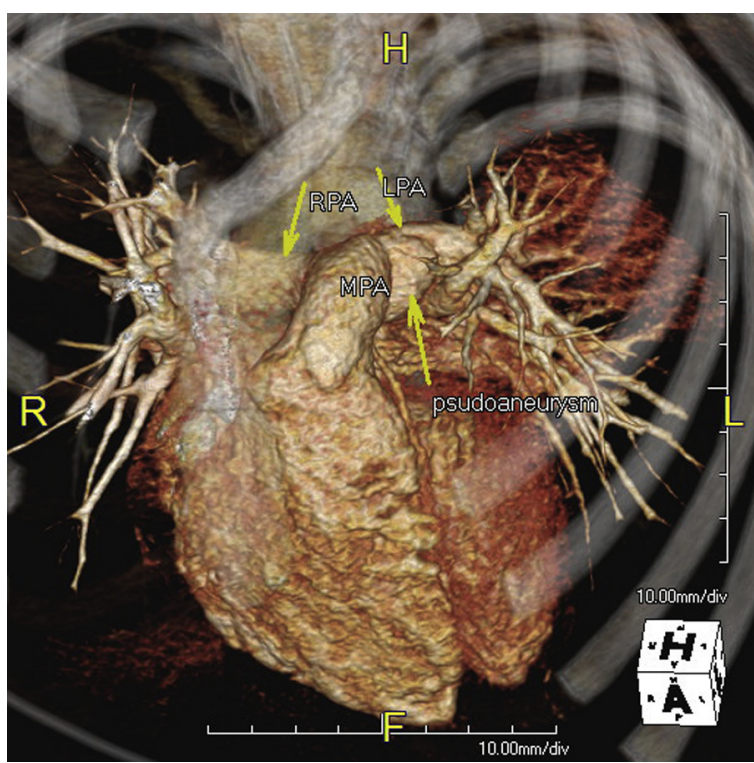

FIGURE 1. Gated chest computed tomographic angiogram demonstrating a $1.9 \times 1.1 \times 2.6$-cm contrast-filled outpouching, described as a moderate pseudoaneurysm, at the proximal left pulmonary artery ( $L P A)$, just beyond the bifurcation of the pulmonary arteries. Anteroposterior cranial view. RPA, Right pulmonary artery; $M P A$, main pulmonary artery.

In the catheterization laboratory, femoral arterial and venous access was obtained. With the percutaneous Seldinger technique, a sidearm sheath was placed in the

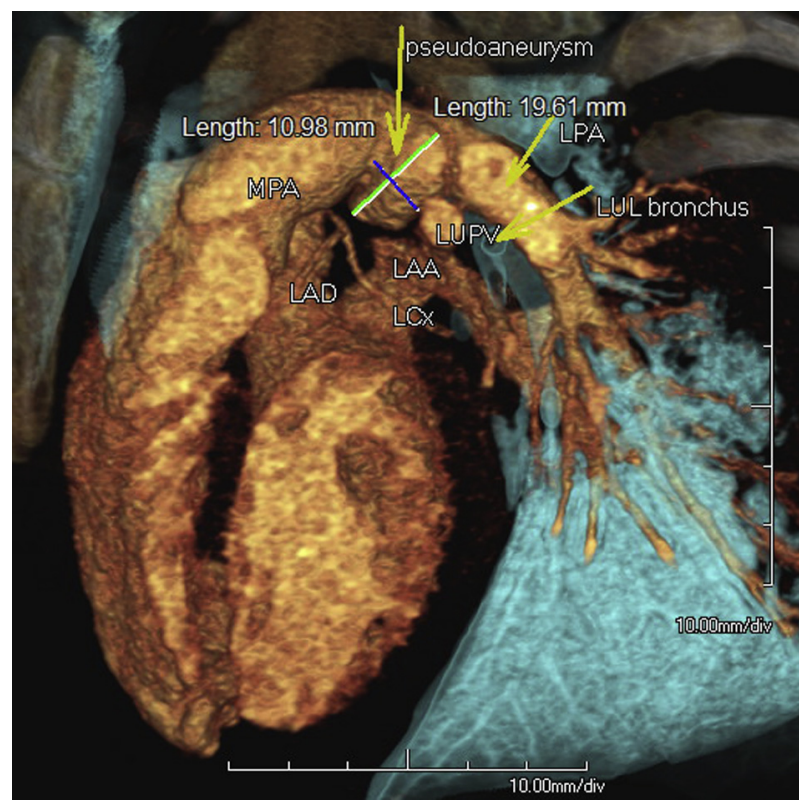

FIGURE 2. Gated chest computed tomographic angiogram demonstrating a $1.9 \times 1.1 \times 2.6$-cm contrast-filled outpouching, described as a moderate pseudoaneurysm, at the proximal left pulmonary artery (LPA), just beyond the bifurcation of the pulmonary arteries. Craniocaudal view. $M P A$, Main pulmonary artery; $L U P V$, left upper pulmonary vein; $L U L$, left upper lobe; $L A A$, left atrial appendage; $L A D$, left anterior descending coronary artery; $L C x$, left circumflex coronary artery.

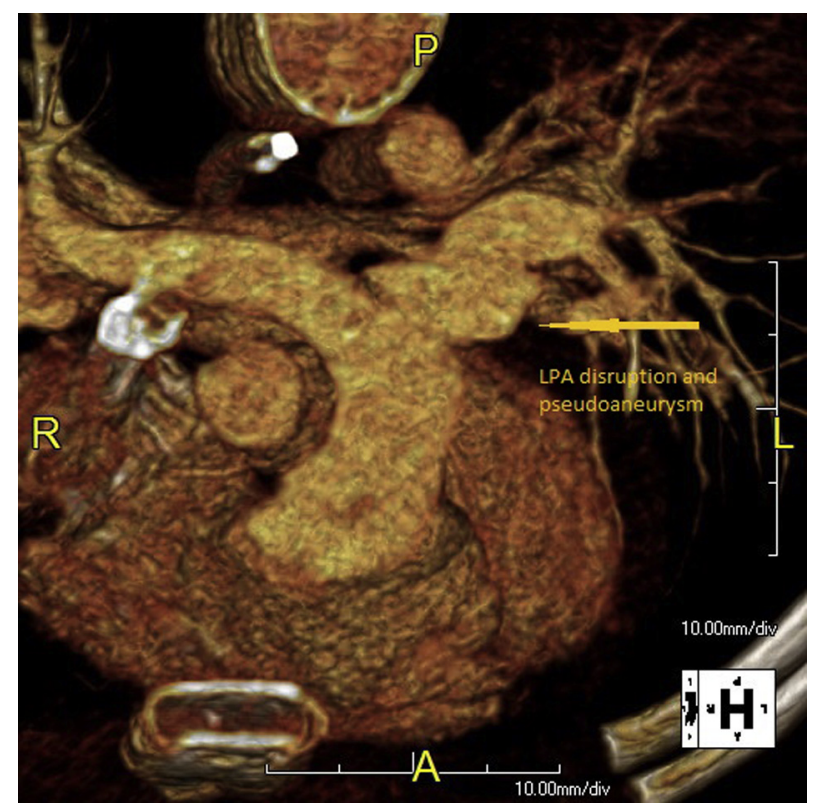

FIGURE 3. Gated chest computed tomographic angiogram demonstrating a $1.9 \times 1.1 \times 2.6-\mathrm{cm}$ contrast-filled outpouching, described as a moderate pseudoaneurysm, at the proximal left pulmonary artery ( $L P A)$, just beyond the bifurcation of the pulmonary arteries. Left-lateral view.

femoral vein. An angiogram was performed in the main pulmonary artery (MPA; Figures 4 and 5). A balloon wedge catheter was advanced into the left pulmonary artery (LPA) and exchanged for a transseptal sheath. A balloon-inballoon angioplasty catheter (BIB; NuMED) with the mounted covered Cheatham platinum stent was advanced through the transseptal sheath to the level of the pseudoaneurysm. The stent was expanded in place, and an angiogram confirming placement was performed. The

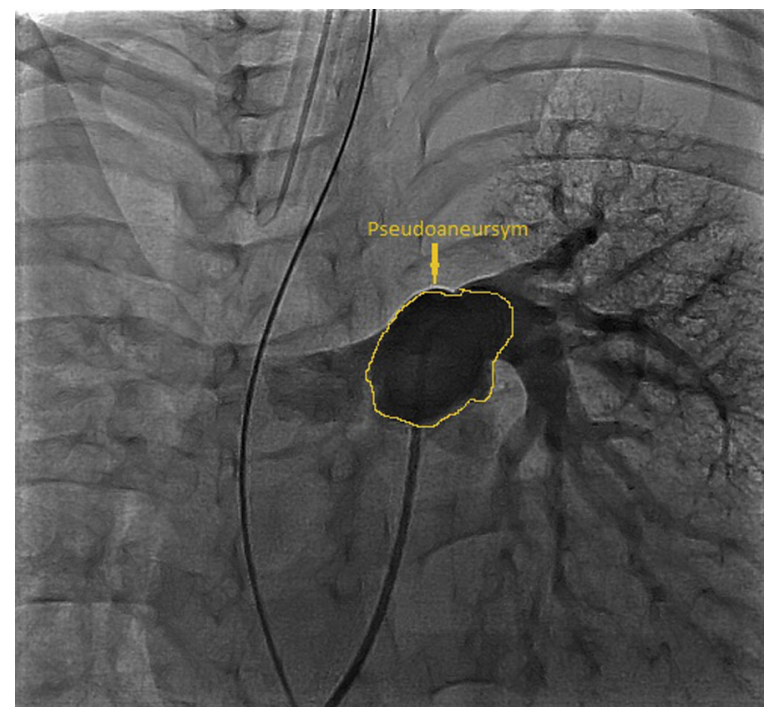

FIGURE 4. Anteroposterior computed tomographic angiogram demonstrating the pseudoaneurysm before intervention in the catheterization laboratory. 


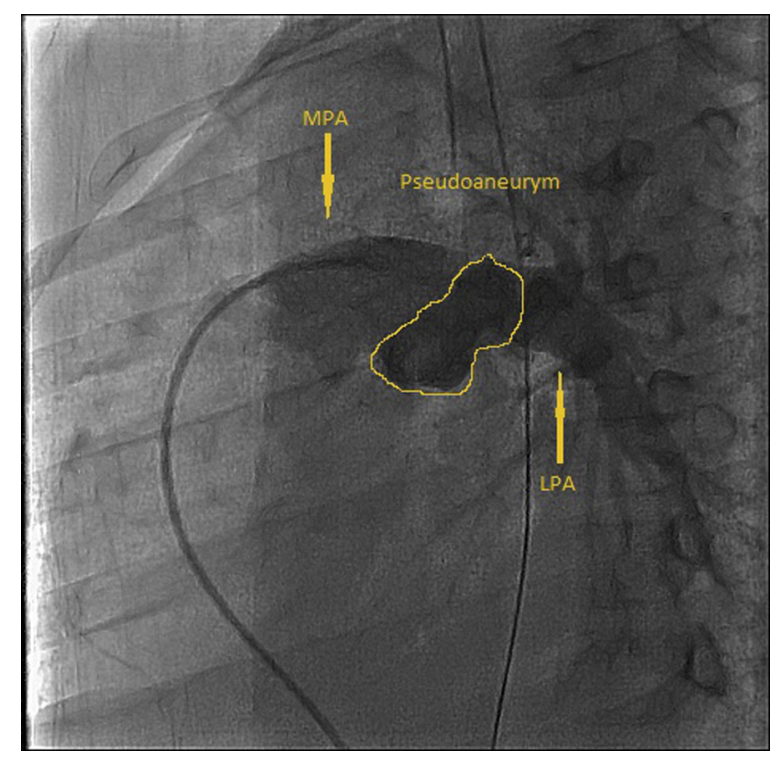

FIGURE 5. Left-lateral computed tomographic angiogram demonstrating the pseudoaneurysm before intervention in the catheterization laboratory. $M P A$, Main pulmonary artery; $L P A$, left pulmonary artery.

filling of the pseudoaneurysm was noted from the MPA between the branch pulmonary arteries (Figure 6). This access into the pseudoaneurysm was noted after the LPA was covered. This catheter was removed, and a second covered stent was used to cover the proximal LPA and extend into the MPA. Angiography confirmed the presence of all LPA branches (Figure 7). The pseudoaneurysm filling

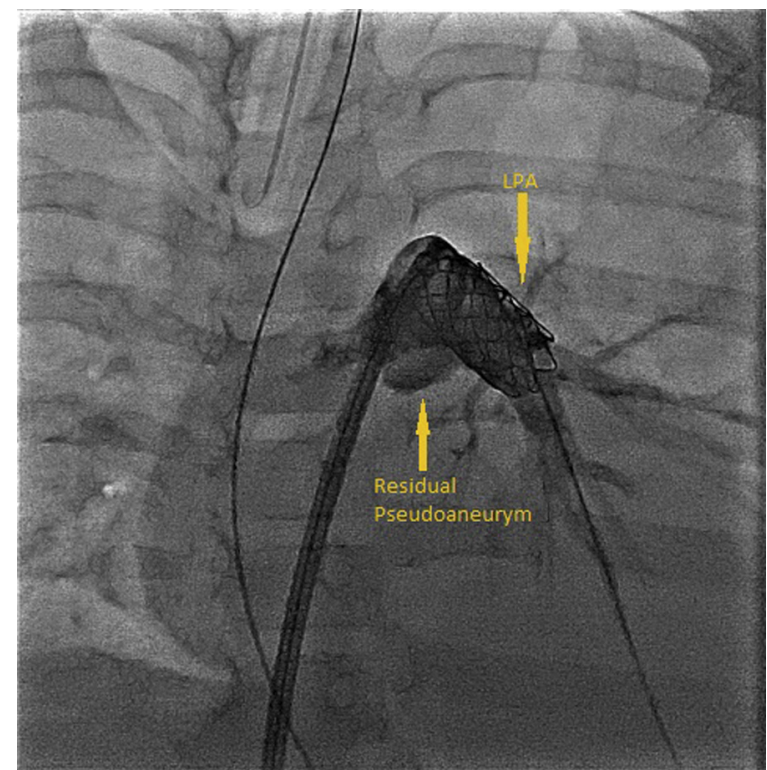

FIGURE 6. Anteroposterior computed tomographic angiogram after the first covered Cheatham platinum stent was placed in the left pulmonary artery $(L P A)$ demonstrates filling of the pseudoaneurysm from the main pulmonary artery, between the branch pulmonary arteries.

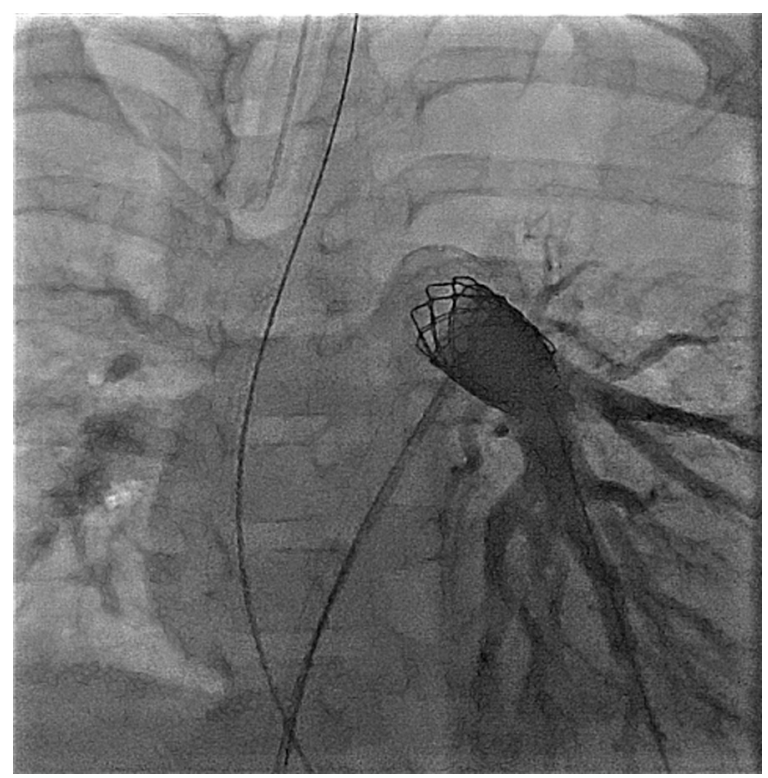

FIGURE 7. Anteroposterior computed tomographic angiogram confirms the presence of all left pulmonary artery branches after the second covered Cheatham platinum stent was placed to cover the left pulmonary artery and extend into the main pulmonary artery.

after the second stent was placed was noted to be present but slower (Figure 8). The stent was dilated proximally to obtain apposition to the MPA wall, which was confirmed by poststent angiograms (Figure 9). The pseudoaneurysm filling was no longer seen after stent dilation.

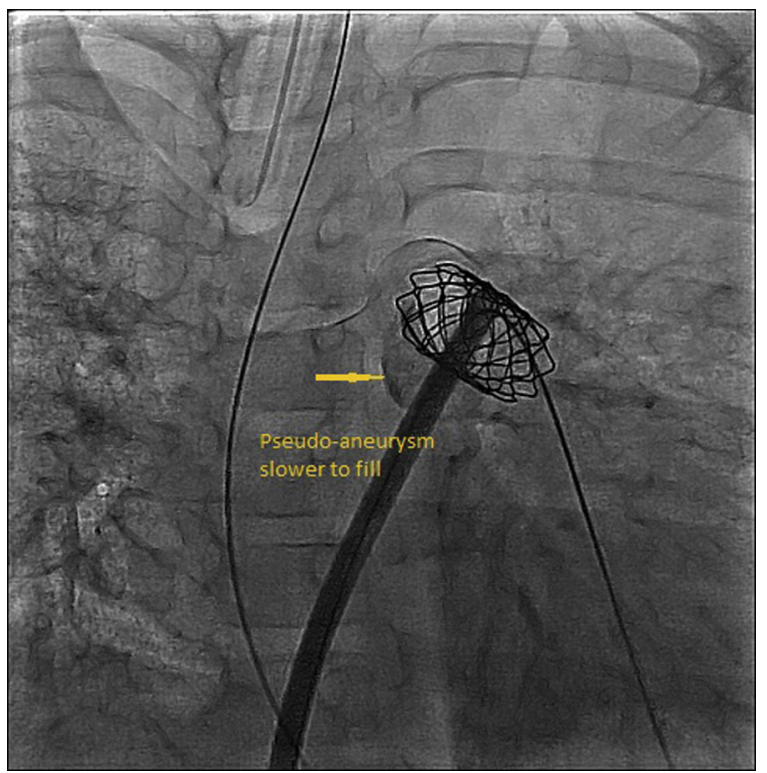

FIGURE 8. Anteroposterior computed tomographic angiogram shows pseudoaneurysm filling to be present but slower after the second stent was placed. 


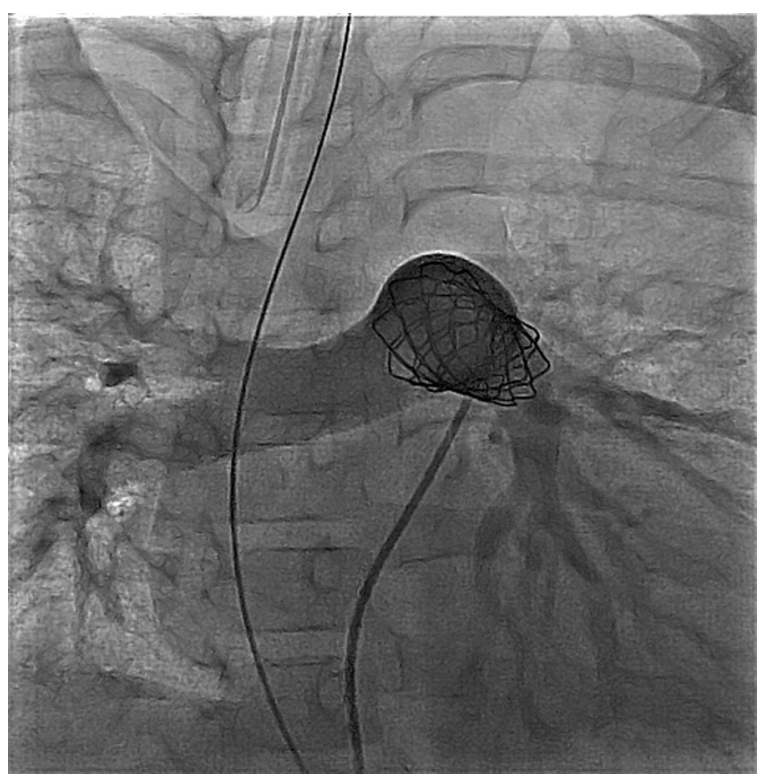

FIGURE 9. Anteroposterior computed tomographic angiogram after the second stent was proximally dilated to obtain apposition to the main pulmonary artery wall shows no pseudoaneurysm filling.

The patient was transported in stable condition to the pediatric intensive care unit for further management. He underwent a follow-up echocardiogram 1 week later, which showed an unobstructed LPA stent and stable, trivial pericardial effusion. The patient's postcatheterization course was uncomplicated, and he was discharged 2 weeks later. At a 2-month follow-up visit, his computed tomographic scan demonstrated interval placement of an LPA stent without evidence of complication or distal filling defect and normal pulmonary arterial perfusion. The patient will be followed up every 6 months.

\section{DISCUSSION}

Because of the rare nature of traumatic pediatric PAPs, an algorithm for their treatment does not exist. Many have diverted to an endovascular approach, because open surgery carries higher morbidity and mortality risks; these patients have frequent comorbidities and coinciding systemic coagulation contraindications. Balloon-expandable covered Cheatham platinum stents can be delivered through sheaths as small as $14 \mathrm{~F}$, allowing their implantation in a patient as small as $20 \mathrm{~kg}$ (our patient weighed $40 \mathrm{~kg}$ ). ${ }^{5}$ Additional advantages to treatment with covered stents include the ability to redilate them to parallel the inevitable pulmonary artery growth and a shorter overall recovery time. Disadvantages to this treatment, however, include the risk of pulmonary artery rupture with initial balloon expansion, along with the lack of comprehensive long-term outcome data.

\section{CONCLUSIONS}

Percutaneous implantation of balloon-expandable covered stents may be a safe and efficacious alternative to open surgical repair and other endovascular approaches for the treatment of traumatic PAPs in the pediatric population.

\section{References}

1. Krokidis M, Spiliopoulos S, Ahmed I, Gkoutzios P, Sabharwal T, Reidy J. Emergency endovascular management of pulmonary artery aneurysms and pseudoaneurysms for the treatment of massive haemoptysis. Hellenic J Cardiol. 2014; 55:204-10.

2. Hannan RL, Miyaji K, Burke RP, Zahn EM. Endovascular stent graft treatment of a pulmonary artery pseudoaneurysm. Ann Thorac Surg. 2001;71:727-9.

3. Wilson N, McLeod K, Hallworth D. Images in cardiology. Exclusion of a pulmonary artery aneurysm using a covered stent. Heart. 2000;83:438.

4. Park A, Cwikiel W. Endovascular treatment of a pulmonary artery pseudoaneurysm with a stent graft: report of two cases. Acta Radiol. 2007;48:45-7.

5. Sandgren T, Sonesson B, Ahlgren R, Länne T. The diameter of the common femoral artery in healthy human: influence of sex, age, and body size. J Vasc Surg. 1999;29:503-10. 